\title{
Einige naturgeographische Grundzüge Nordfinnlands
}

Matti Häkkilä*

\section{Allgemeines}

Unter dem Begriff «Nordfinnland» wird gewöhnlich das Gebiet der Provinzen (finnisch: lääni) Oulu und Lappland verstanden, ein Gebiet, dessen GröBe 130345 km², 47,6\% der Gesamtfläche Finnlands ausmacht, dessen Einwohnerzahl aber nur 612000 beträgt, was $14 \%$ der Gesamtbevölkerung Finnlands entspricht. Zwei Drittel der Einwohner Nordfinnlands wohnen im südlichen Teil des Gebietes, in der Provinz Oulu. Hier beträgt die Bevölkerungsdichte 7,4 Einwohner $/ \mathrm{km}^{2}$, in der Provinz Lappland 2,3 Einwohner $/ \mathrm{km}^{2}$, während sie für ganz Finnland 15,1 ausmacht. Vergleichshalber sei erwähnt, daß die Bevölkerungsdichte in der Provinz Uusimaa, der südlichsten Finnlands, in der auch die Hauptstadt Helsinki liegt, 95,6 Einwohner $/ \mathrm{km}^{2}$ beträgt. Weiterhin ist zu bemerken, daß die Hälfte der Einwohner Nordfinnlands in Städten wohnt, weshalb die Bevölkerungsdichte auf dem Lande in der Provinz Oulu nur 2,2 Einwohner $/ \mathrm{km}^{2}$ und in der Provinz Lappland nur 1,1 Einwohner $/ \mathrm{km}^{2}$ ausmacht. Deshalb gehört auch ein großer Teil Nordfinnlands zum sogenannten "Naturfinnland» (im Gegensatz zum «Kulturfinnland»).

\section{Gesteinsgrundlage und Höhenverhältnisse}

Finnland gehört in seiner Gesamtheit zum fennoskandischen Grundgebirge, des von den harten kristallinen Gesteinen des ältesten oder archaischen Erdzeitalters aufgebaut wird. In Nordfinnland finden sich jedoch nicht so viele Granitgebiete wie in Südfinnland. In Westlappland, nördlich von Rovaniemi, und in den Regionen östlich von Kemijärvi im Südteil der Provinz Lappland kommt Granit sehr häufig vor. In Lappland treten auch weiter nördlich noch ausgedehnte Granitgebiete auf. In der Provinz Oulu besteht das Küstengebiet, das sich von Oulu nach Süden erstreckt, ebenfalls aus Granit oder aus ihm verwandten Tiefengestei-

\footnotetext{
* Herr Matti Häkkilä, Assistent am Geographischen Institut der Universität Oulu, studierte 1970 während vier Monaten am Geographischen Institut der ETH Zürich. Er war Stipendiat der Schweizerischen Vereinigung der Freunde Finnlands.
}

nen. Eine Besonderheit bildet südlich des Flusses Oulujoki eine ungefähr $500 \mathrm{~m}$ mächtige Sedimentablagerung von Tonschiefern und Sandsteinen, die Muhos-Formation. In der Hauptsache umfaßt der Grund Nordfinnlands jedoch in der sogenannten karelischen Faltungsphase entstandene Gesteine, unter denen verschiedene kristalline Schiefer vorherrschen, wie Granitgneis, Granulit, Quarzit, Phyllit, Glimmerschiefer, Migmatit, Glimmergneis usw. Im Grundgebirge Finnlands gibt es auch Erzvorkommen, obwohl in Finnland bisher nicht so bedeutende entdeckt worden sind wie im Nachbarland Schweden. Eine verstärkte Erzsuche, bei der die ganze Bevölkerung zur Mitarbeit aufgerufen wurde, hat in den letzten Jahren die Zahl der Gruben und die Erzförderung bedeutend vergrößert.

Im finnischen Bergbau hat Nordfinnland eine starke Stellung inne. Alle wichtigen Eisenerzgruben des Landes befinden sich in dieser Region: z.B. in Lappland die Eisengruben von Kolair, Kärväsvaara und Raajärvi. Von besonderer Bedeutung ist diejenige von Otanmäki in der Gemeinde Vuolijoki in der Provinz Oulu. Die jährliche Eisenerzförderung Finnlands überschreitet eine Million Tonnen.

In der Gemeinde Vihanti (Provinz Oulu) befindet sich die Grube von Alpuna, die vor allem Zink fördert. Sie ist Europas größte Zinkgrube. Als Nebenprodukt wird hier auch Bleierz gewonnen. Da die Nickelgruben im Gebiet von Petsamo nach dem Krieg verlorengingen, ist der Mangel an Nickel in Suomi spürbar geworden. Immerhin konnte unlängst u. a. in Nivala, im Südteil der Provinz Oulu, eine neue Nickelgrube eröffnet werden. In der Gemeinde Pyhäjärvi, ebenfalls in der Provinz Oulu, liegt eine bedeutende Schwefelkies- und Kobaltgrube. In der Nähe der Stadt Kemi (Lappland) wird Chromerz gefördert. Die bereits erwähnte Grube von Otanmäki fördert neben Eisenerz u. a. auch Vanadium. Auch in der Gewinnung von Industriemineralien ist Nordfinnland von Bedeutung. So wird beispielsweise im südlichen Westlappland Kalkstein, in der Umgebung des Oulujärvi-Sees Talk abgebaut. Das Relief Nordfinnlands wirkt zumeist einförmig eben, wenn man es mit jenem der Schweiz vergleicht. Von der fast völlig flachen Küstenzone am Bottnischen Meerbusen steigt das Land ziemlich gleichmäßig nach Osten und Norden an. Hie und da überragen sanft ansteigende Erhebungen die Landschaft, die gewöhnlich aus Quarzit bestehen. 


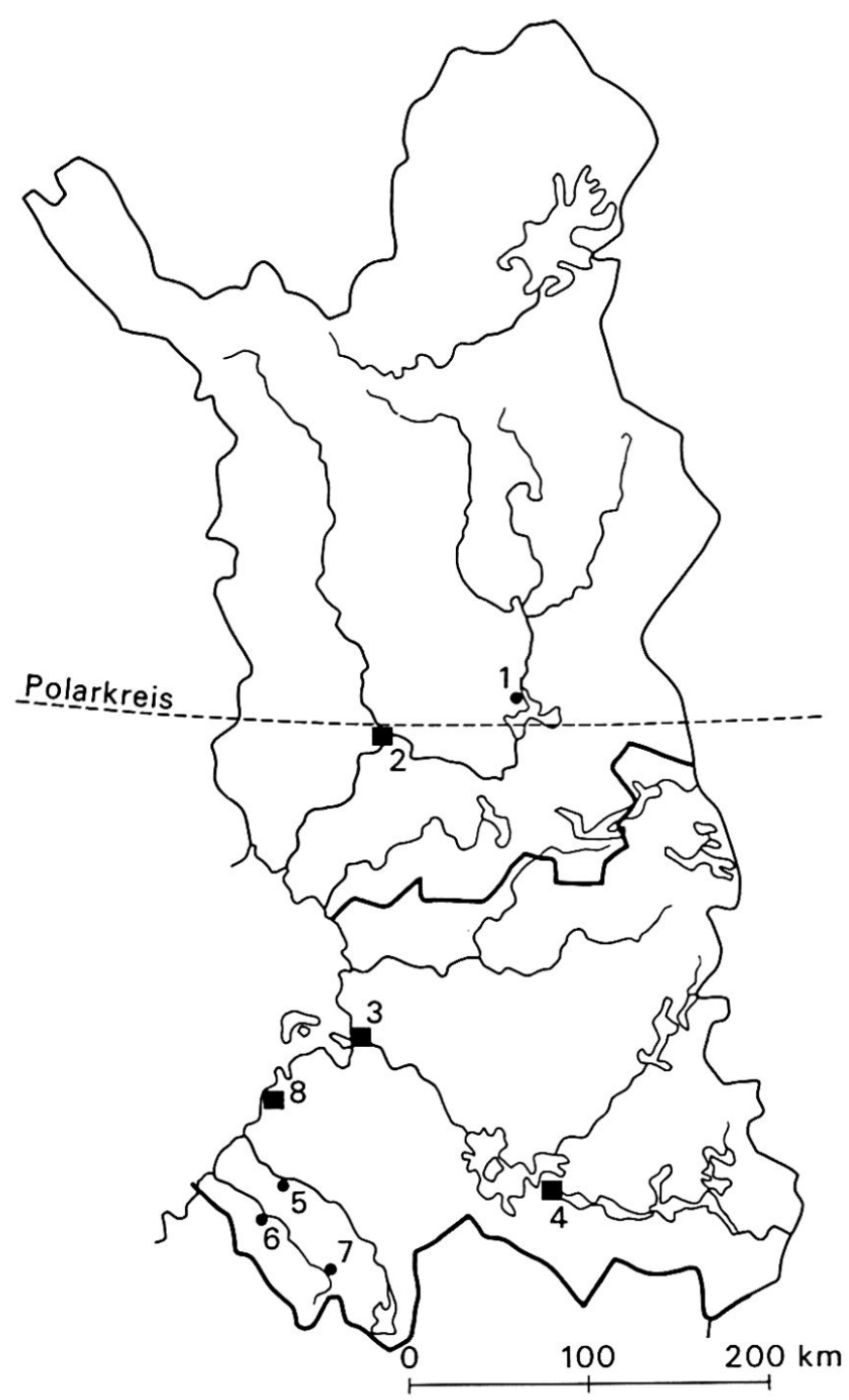

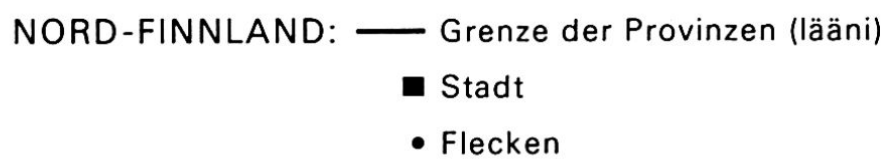

(1=Kemijärvi, 2 =Rovaniemi, $3=$ Oulu, $4=$ Kajaani

$5=$ Oulainen, $6=$ Ylivieska, $7=$ Haapajärvi, $8=$ Raahe)

Im Südteil dieses Gebietes sind diese Anhöhen noch gänzlich mit Wald bedeckt; sie werden auf Finnisch "vaara» genannt. In Lappland sind die Kuppen völlig kahl; auf Finnisch heißen sie hier «tunturi». Weil ihr Gestein härter ist als das ihrer Umgebung, hat dieses Gelände der im Laufe von Jahrmillionen wirkenden Abtragung besser widerstanden. In der Provinz Oulu bleiben die Erhebungen (Isosyöte, Pyhitys, Vuokatti, Valtavaara, Iivaara usw.) gewöhnlich unter $500 \mathrm{~m}$ Höhe. Die Höhe der "tunturis» in Lappland beträgt gewöhnlich 500 bis $800 \mathrm{~m}$ mit Ausnahme einiger Gipfel im nordwestlichen «Zipfel» Finnlands, die über $1000 \mathrm{~m}$ aufragen (Haltiatunturi $1324 \mathrm{~m}$, höchster Berg Finnlands; Saana, Kahperusvaara usw.), die aber geologisch bereits zu einem völlig anderen Bereich, zum Skandinavischen Gebirge, gehören.
Nordfinnland, wie überhaupt ganz Fennoskandien, gehört geologisch zu den «ruhigsten» Gebieten des Erdballs. An endogenen Kräften macht sich in Finnland lediglich die Landhebung bemerkbar; sie hat im Laufe der Zeit bedeutende Veränderungen sowohl in der Natur als in der Kulturlandschaft verursacht und wirkt heute noch weiter. Die Landhebung ist eine Folge der eiszeitlichen Vergletscherung. Nach der Befreiung vom Druck der Eismassen hebt sich die Erdoberfläche allmählich, um wieder in den vor den Eiszeiten herrschenden Gleichgewichtszustand zu gelangen. In Finnland ist die Landhebung im mittleren Teil des Bottnischen Meerbusens am stärksten; sie beträgt in hundert Jahren ungefähr $90 \mathrm{~cm}$. Die Intensität der Landhebung verringert sich nach Norden und Nordosten und beträgt beispielsweise im Gebiet von Kuusamo weniger als $70 \mathrm{~cm}$ in hundert Jahren.

\section{Der Boden}

Der Felsgrund von Nordfinnland wird fast überall von einer Erdschicht bedeckt. Die Abgrenzung gegenüber der Gesteinsunterlage ist im allgemeinen sehr klar. Nur an Stellen, wo der Grund stark verwittert oder wo die Abschürfung durch das Inlandeis gering war, wie stellenweise in Lappland, verläuft die Grenze zwischen dem lockeren Boden und dem liegenden Gestein in Abstufungen. Den größten Teil Nordfinnlands bedecken die während der letzten Vereisungsphasen entstandenen Moränen, Sande und Lehme. Uber ihnen hat sich später Torf gebildet. Die Mächtigkeit der Moränenablagerungen beträgt durchschnittlich 3-5 m.

Die Grundmoräne bedeckt den Felsengrund als dünne, oft stark zusammengedrückte Decke und nivelliert die Unebenheiten. In Richtung der Gletscherbewegung verlaufen längliche und schmale Moränenhügel mit gelegentlichem Felskern, die Drumlins. Sie sind in Nordfinnland reichlich anzutreffen.

Sortierte glaziale Ablagerungen, sogenannte glazialfluviale Schotter, treten viel kleinflächiger auf als Moränen. Die Schmelzwasser des Eises haben den Moräneninhalt sortiert, die Bestandteile, die gröber als Sand sind, bilden das hauptsächliche Material der Oser (finnisch: harju), deren Richtung ungefähr der Rückzugsrichtung des Gletschereises entspricht. Schmale sich weit hinziehende, oft sogar mehr als hundert Kilometer lange Oszüge sind in den verschiedenen Teilen Nordfinnlands reichlich anzutreffen, am meisten vielleicht in Mittellappland. Der Os-Sand gilt als vorzügliches Straßenbaumaterial.

Die feineren glazialfluvialen Materialien, die das Schmelzwasser des Eises aus der Moräne wusch, wie Lehme, Tone und Sande, sind weiter transportiert worden und haben sich schließlich am Grunde des 


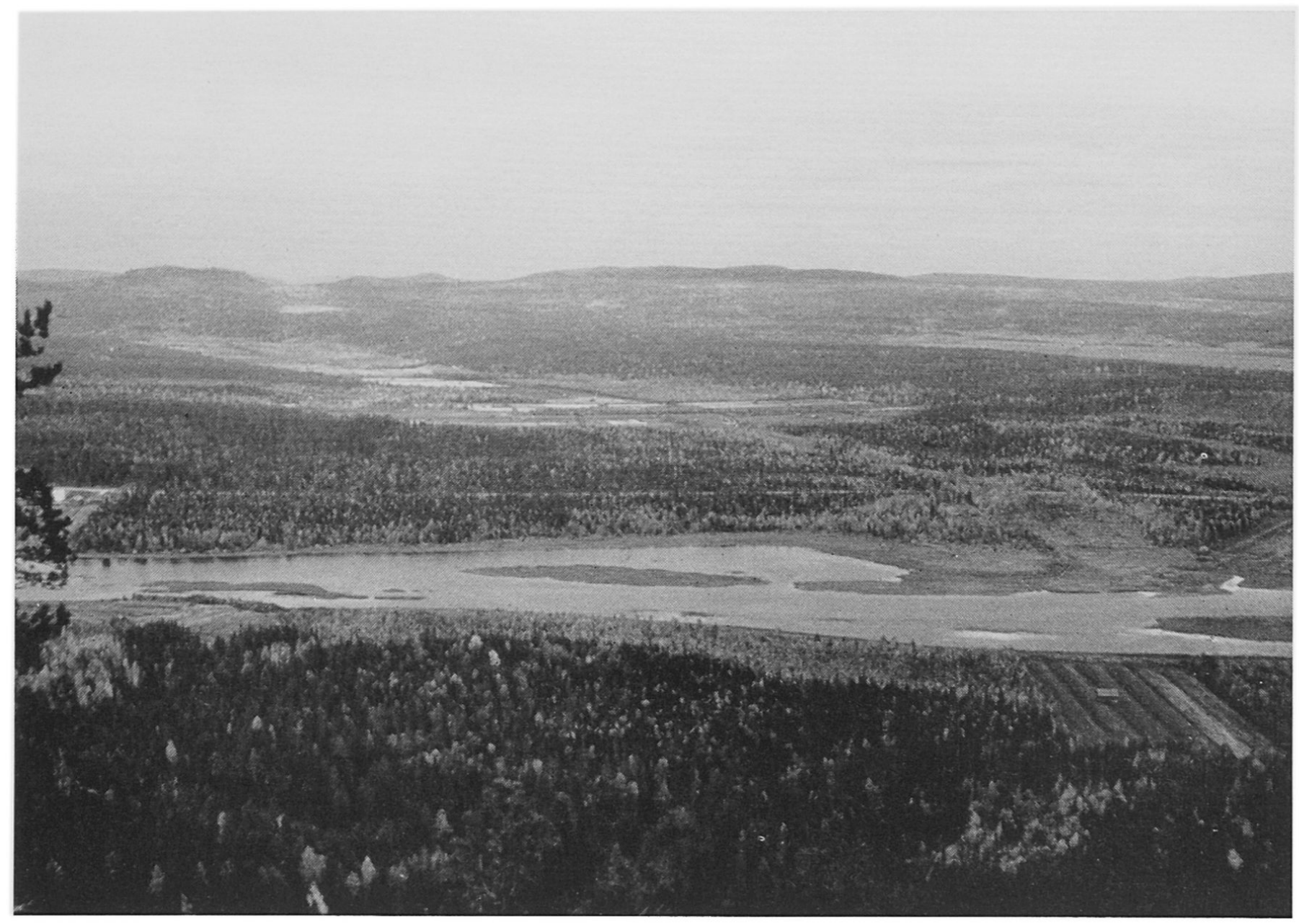

Abbildung 1. Landschaft in Aavasaksa. Wälder, Moore und Flüsse sind die Hauptelemente der Landschaft im südlichen Lappland. Photo M. Häkkilä

Meeres oder der Seen abgelagert, von wo sie anläßlich der Landhebung über den Wasserspiegel gehoben wurden. Als Ackerböden sind sie jetzt von sehr großer Bedeutung. Lehm- und Sandfelder treten besonders im Küstengebiet des Bottnischen Meerbusens, an den Ufern der großen Seen und längs der Flüsse auf. An den Flußufern liegen auch postglazial entstandene, vom Fluß bei Hochwasser aufgeschüttete Lehm- und Sandfelder.

$\mathrm{Zu}$ den jüngsten Ablagerungen gehören die Flugsandfelder. Sie treten vor allem an der Küste auf, wo der vom Meer her wehende Wind den Sand trocknet und ihn zu Strandwällen aufbaut. Unbewachsene Flugsandanwehungen gibt es auch in Lappland an Stellen, wo starker Wind in Gebieten schwacher Vegetation auftritt. Das bekannteste Flugsandfeld Nordfinnlands liegt an der Küste des Bottnischen Meerbusens im Gebiet von Kalajoki: es bildet heute ein beliebtes Touristenziel.

Wesentlich häufiger als die rezenten Wanderdünen sind in Nordfinnland fossile, durch Pflanzenwuchs gebundene Dünen. Auch sie sind unter dem Einfluß des Windes aus Uferablagerungen entstanden; da sich das Ufer infolge der Landhebung verschob, wurden sie von der Vegetation rasch überzogen und verfestigt.

Ein bedeutender Teil der Böden Nordfinnlands wird von Torfablagerungen überdeckt. Da es in Nordfinnland ausgedehnte ebene Gebiete gibt und der Mineralboden kaum wasserdurchlässig ist, sind die Voraussetzungen für die Versumpfung günstig. Die am meisten versumpften Gebiete liegen in einer breiten Zone längs der Küste des Bottnischen Meerbusens, wo Moore mehr als $60 \%$ der Oberfläche ausmachen. Danach folgt, hinsichtlich des Grades der Versumpfung, Mittel-Lappland; im Gebiet zwischen den Flüssen Tornionjoki, Ounasjoki, Kitinen, Luiro und Tenniöjoki beträgt der Anteil der Moore an der Oberfläche 50-60\%. Am wenigsten Moore gibt es in Inari und Utsjoki, ihr Anteil an der Oberfläche beträgt nur 10-20\%. Moore haben die Tendenz, das ohnehin fast ebene Relief noch mehr zu nivellieren. Ein bedeutender Teil der Moore Nordfinnlands ist mit Drainagegräben versehen und aufgeforstet worden. Man hat auch begonnen, den 


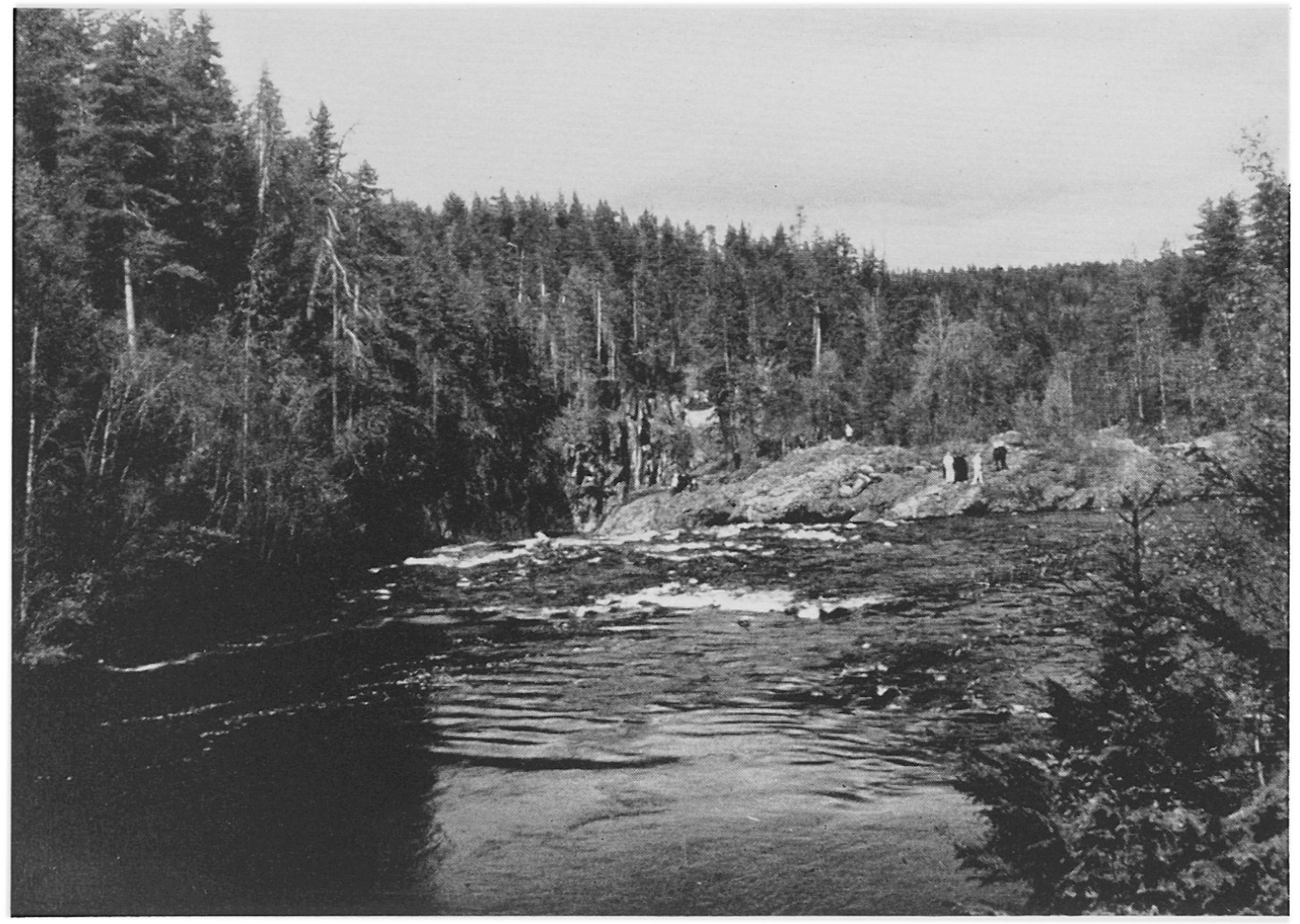

Abbildung 2. Der Fluß Oulankajoki im Nationalpark Oulanka in Kuusamo, Nordostfinnland. Photo M. Häkkilä

Torf abzubauen und als Brenn- oder Düngemittel zu verwenden.

\section{Das Klima}

Trotz der nördlichen Lage ist das Klima Nordfinnlands - im Vergleich zu vielen anderen Gebieten, die auf derselben geographischen Breite liegen ziemlich mild. Der sich bis zum Eismeer erstrekkende Golfstrom mildert es; zusätzlich geben der Bottnische Meerbusen und das Weiße Meer, wenn sie nicht zugefroren sind, dem hier sonst vorherrschenden Kontinentalklima einen maritimen Anstrich. Der Nordteil des Bottnischen Meerbusens ist im Winter während sechs bis sieben Monaten vereist, die Seen im Küstengebiet des Bottnischen Meerbusen während ungefähr 7 und in Nordlappland während ungefähr 8 Monaten. Die Schneedecke bleibt im Küstengebiet des Bottnischen Meerbusens etwa fünfeinhalb Monate lang liegen. Nord-Lappland ist sogar durchschnittlich während 7 Monaten von Schnee bedeckt. Die Jahresdurchschnittstem- peratur beträgt in den südlichen Teilen des Gebietes $2-3^{\circ} \mathrm{C}$ und in den nordwestlichen Teilen ungefähr $-1^{\circ} \mathrm{C}$. Die jahreszeitliche Temperaturschwankung beträgt im Küstengebiet des Bottnischen Meerbusens $26-27^{\circ} \mathrm{C}$ und im Gebiet der Gemeinden Enontekiö, Kittilä, Sodankylä und Savukoski ungefähr $28^{\circ} \mathrm{C}$. Diese Region stellt den kontinentalsten Teil Nordfinnlands dar. Im Winter kann dort die Temperatur unter $-40^{\circ} \mathrm{C}$ sinken, im Sommer aber auf mehr als $30^{\circ} \mathrm{C}$ steigen. Im Winter bleibt die Lufttemperatur in Lappland durchschnittlich 200 Tage ganz unter $0^{\circ} \mathrm{C}$, in Oulu 165 Tage lang, in Helsinki aber beispielsweise nur während 120 Tagen. Die Zeit, in der die Tagesdurchschnittstemperatur $+5^{\circ} \mathrm{C}$ oder mehr beträgt, dauert in den südwestlichen Teilen der Provinz Oulu 150 Tage und verkürzt sich in Enontekio und Utsjoki auf 120 Tage. Die jährliche Niederschlagsmenge beträgt in den östlichen Teilen der Provinz Oulu mehr als $600 \mathrm{~mm}$ und verringert sich nach Westen und Norden. In Utsjoki liegt sie bereits unter $400 \mathrm{~mm}$. In der Provinz Lappland fällt fast die Hälfte des Niederschlags als Schnee. Ungefähr 


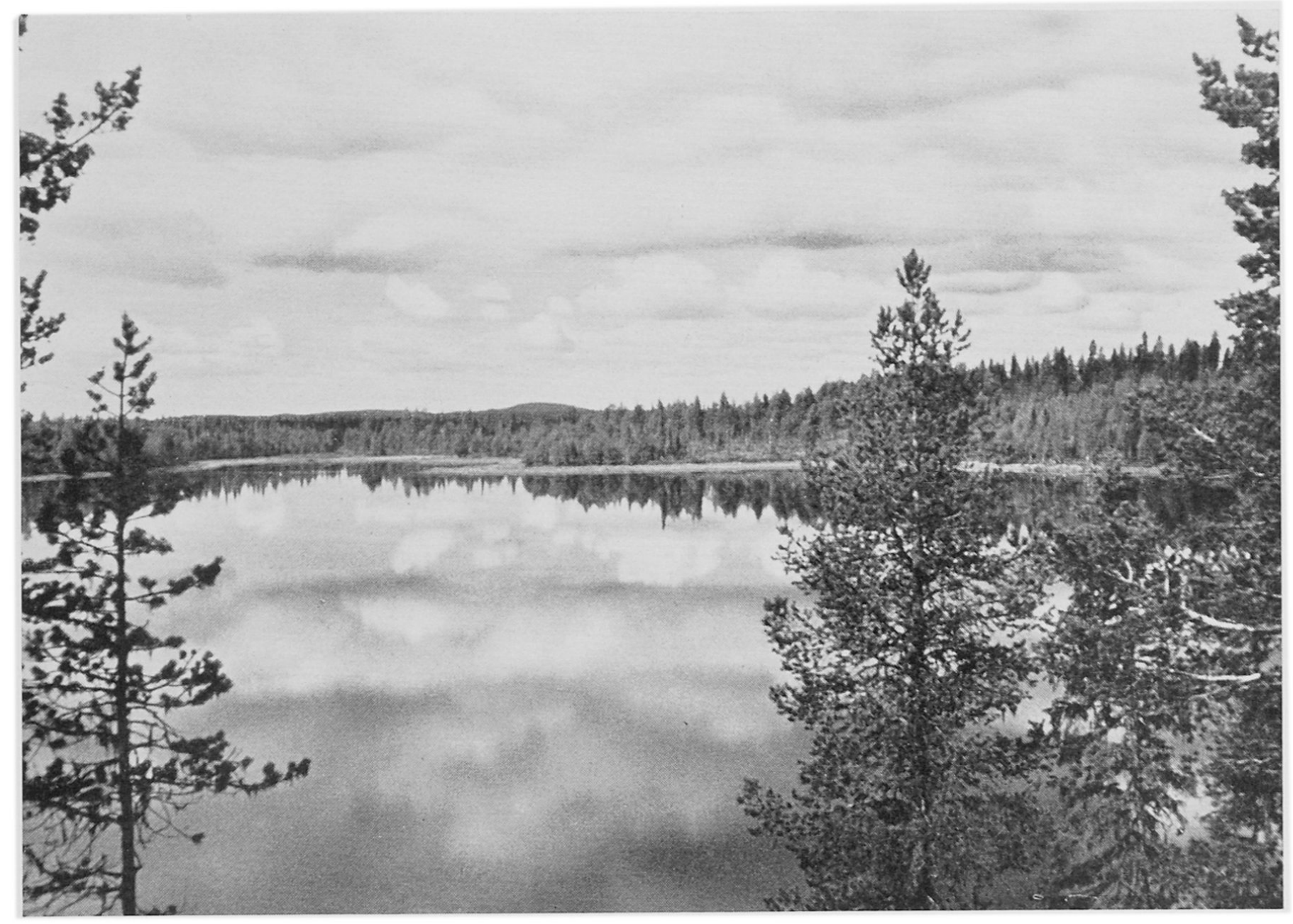

Abbildung 3. Eine Seelandschaft in Kuusamo, Nord ostfinnland. Photo M. Häkkilä

die Hälfte der jährlichen Niederschlagsmenge fällt im Zeitraum Mai-September, ein bedeutender Teil davon als Regen am Ende dieses Zeitraumes. Obwohl in Nordfinnland die absolute Regenmenge gering ist, ist ihr Feuchtigkeitseinfluß groß, weil zufolge der niedrigen Temperaturen auch die Verdunstung klein ist.

\section{Die Lichtverhältnisse}

In Nordfinnland ist der Wechsel in der Tageslänge im Verlauf eines Jahres bedeutend. Im Sommer, wenn die Sonne am höchsten steht, währt in Rovaniemi der ununterbrochene "Mittsommertag" 20 Tage, in Sodankylä ungefähr drei Wochen, im Gebiet von Muonino fast zwei Monate und in Utsjoki mehr als 70 Tage. Im Winter entspricht diesen langen Sommertagen in Lappland eine ebenso lange Zeit der Winterdunkelheit (die auf Finnisch «kaamos» heißt). In Wirklichkeit ist sie jedoch etwas kürzer. In Utsjoki erhebt sich die Sonne während sieben Wochen nicht über den Horizont;
Muoni zählt 23 sonnenlose Tage, und in Sodankylä gibt es noch einen sonnenlosen Tag. Gegen Mittag spiegelt sich allerdings in der Luft der Widerschein der Sonne, selbst wenn sie sich nicht über den Horizont erhebt. Die Dunkelheit macht um die Mittagszeit einem mehrstündigen Dämmerlicht Platz, und am südlichen Horizont bietet sich oft ein farbenfrohes Lichterspiel. Die Winterdunkelheit wird zusätzlich noch durch Nordlichter, durch Mondschein und durch die Reflektion des Schnees gemildert.

Der lange Sommertag ist für die nordfinnische Landwirtschaft von großer Bedeutung. Die Kulturpflanzen können auch in den hellen Nächten assimilieren und dadurch auch trotz des kurzen Sommers zur Reife gelangen.

\section{Die Gewässer}

Obwohl Finnland das «Land der tausend Seen» genannt wird, kann sein Norden doch nicht als besonders seenreiches Gebiet bezeichnet werden. Da 


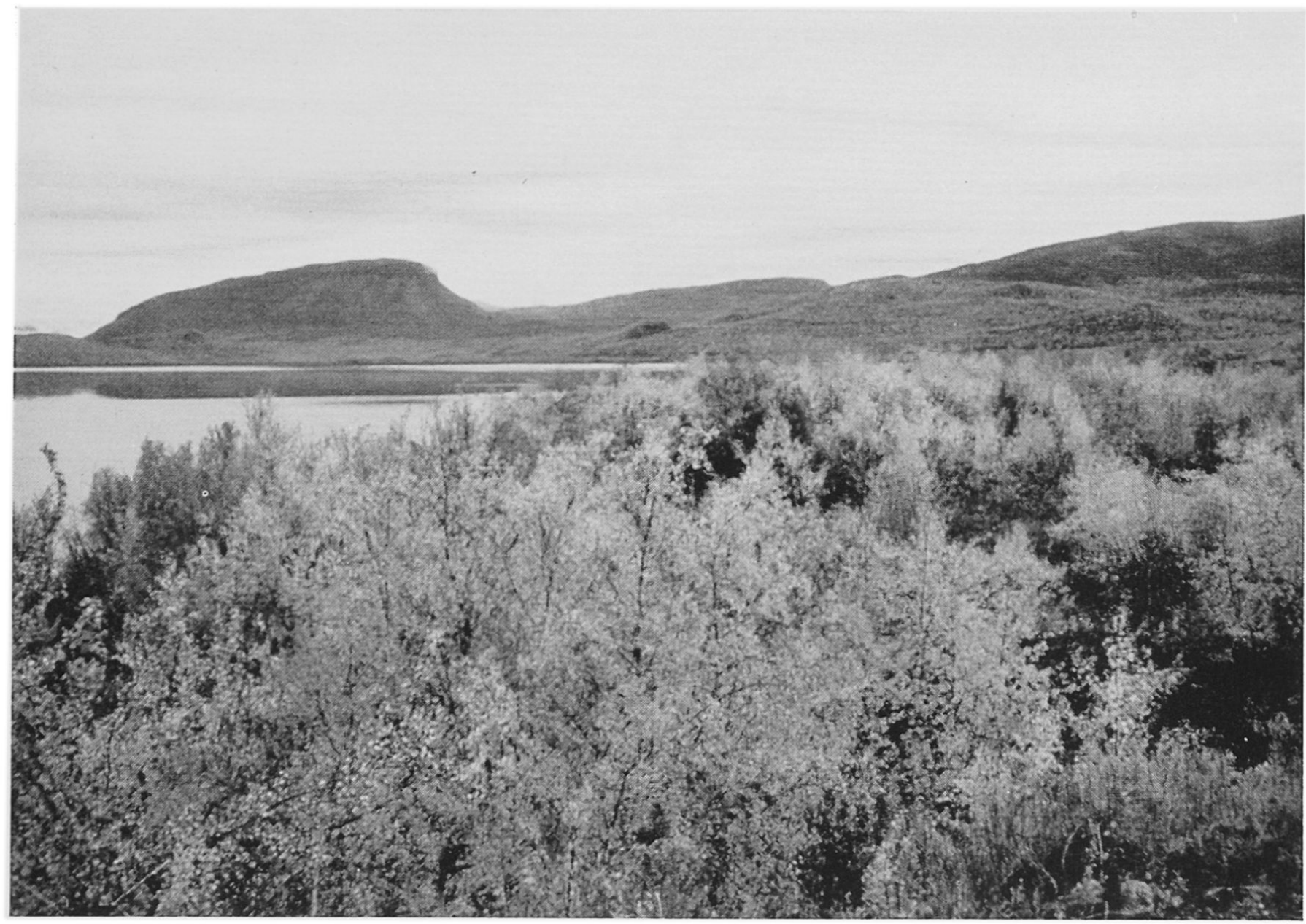

Abbildung 4. Birken-Buschwald in Nordlappland. Im Hintergrund der Saanaberg, $1029 \mathrm{~m}$ ü. M. Photo M. Häkkilä

es eben ist, gibt es hier kaum Einsenkungen, die als Seebecken dienen können. Dazu kommt, daß sich der größte Teil des Gebietes zum Bottnischen Meerbusen neigt und dort keine wasserstauenden Schwellen vorkommen. Seen und Flüsse sind am spärlichsten im Küstenbereich des Bottnischen Meerbusens und in weiten Regionen Nordlapplands, wo ihr Anteil an der Oberfläche sogar unter $5 \%$ liegt. In Kainuu und in Nordostfinnland (Gebiet von Kuusamo) sowie in der Gemeinde Inari in Lappland nehmen die Wasserflächen dagegen stellenweise 15_ $35 \%$ der Gesamtfläche ein. In der Provinz Oulu beträgt der Anteil der Wasserflächen ungefähr 7,8\%, in der Provinz Lappland 5,6\%, in ganz Finnland sogar $10,3 \%$ der Oberfläche.

Die größten Seen Nordfinnlands sind der Fläche nach der Inarinjävi-See, mit $1085 \mathrm{~km}^{2}$ und der Oulujärvi-See mit $887 \mathrm{~km}^{2}$. Die finnischen Seen sind für ihre geringe Tiefe bekannt. In der Gegenwart, in der auch in den industrialisierten Gebieten Nordfinnlands die Verschmutzung der Gewässer ein Problem zu werden beginnt, ist dieser Umstand besonders nachteilig. Jedoch sind vor allem in Lappland die Seen noch sauber und klar. Ihre Ufer und Inseln haben in touristischer Hinsicht an Bedeutung zugenommen. Die Seen selber sind für den Fischfang wichtig, der sowohl von der ansässigen Bevölkerung wie auch von Touristen betrieben wird.

In diesem Zusammenhang sei noch erwähnt, daß in den letzten Jahren in Nordfinnland längs den großen Flüssen künstliche Stauseen angelegt worden sind, die vor allem der Regulierung des Wasserhaushalts und für Wasserkraftwerke dienen. Am bekanntesten sind die beiden größten Stauseen Lapplands, das Becken von Lokka (Oberfläche 407 km²) und jenes von Porttipahta (Oberfläche $214 \mathrm{~km}^{2}$ ). Nordfinnland erzeugt den Hauptteil der finnischen Elektroenergie. Fast alle nutzbaren finnischen Wasserfälle sind in den Dienst der Elektrizitätserzeugung gestellt worden.

$\mathrm{Zu}$ Nordfinnland gehören ungefähr $300 \mathrm{~km}$ Meeresküste, nämlich die Nordostküste des Bottnischen 


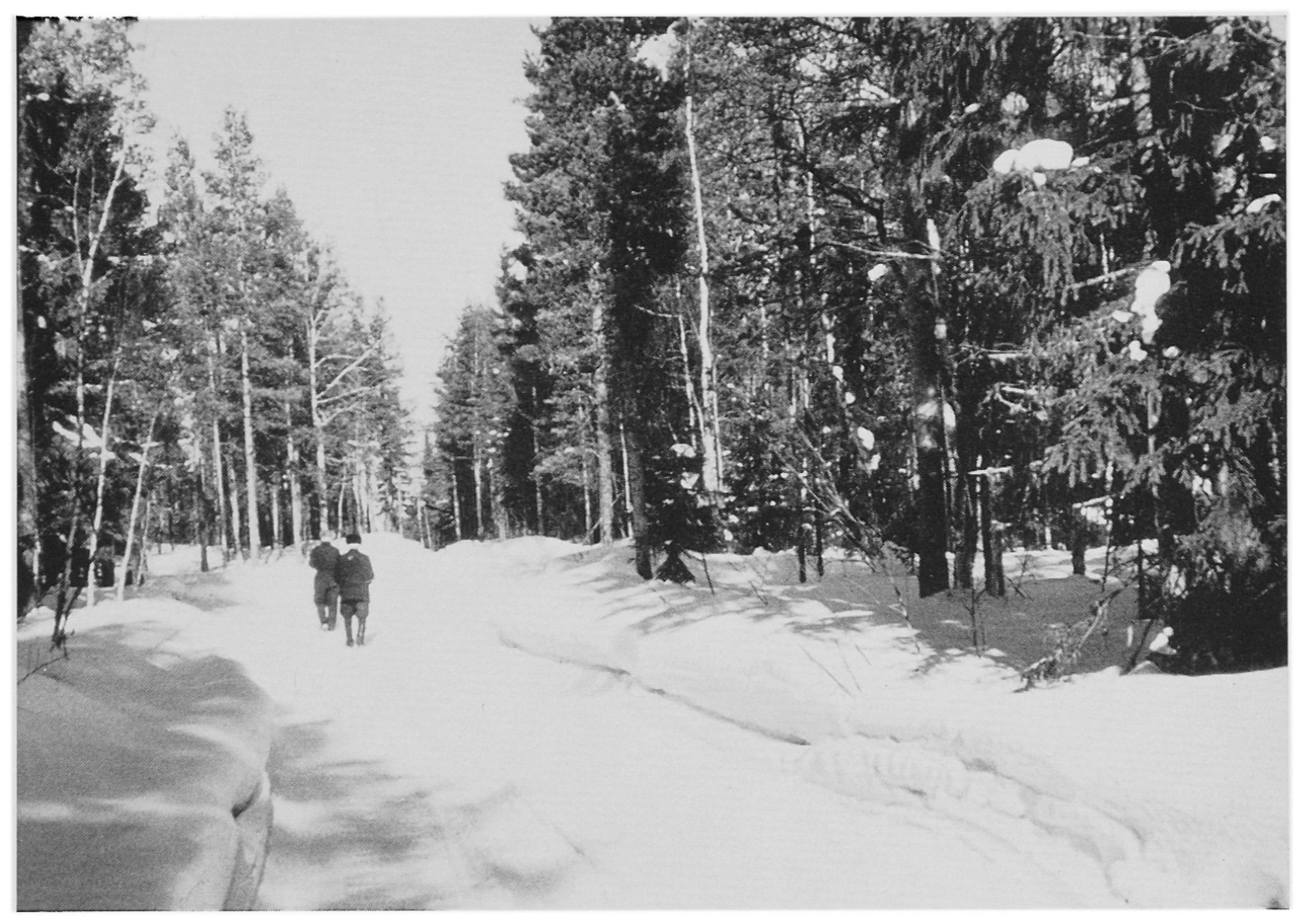

Abbildung 5. Mischwald in Oulainen, im südlichen Teil Nordfinnlands. Photo M. Häkkilä

Meerbusens. In dessen Nordteil ist das Wasser nur noch schwach salzhaltig; der Salzgehalt beträgt ungefähr 3 Promille. Für die Schiffahrt Nordfinnlands hat der Bottnische Meerbusen große Bedeutung; Oulu, Kemi und Raahe sind wichtige Hafenstädte. Zwar ist sein Nordteil im Winter ein halbes Jahr lang zugefroren, die Schffahrt kann jedoch mit Hilfe von Eisbrechern zeitweise aufrechterhalten werden.

\section{Die Vegetation}

Nordfinnland gehört zur polaren Nadelwaldzone. Wald- oder Sumpfvegetation bedeckt den Hauptteil des Gebiets. In vielen Wäldern sind mehr als die Hälfte der Bäume Kiefern. Es folgt die Fichte, deren Anteil an der Fläche der Wälder zwischen 10 und $60 \%$ schwankt. In Nordlappland bildet die Birke die Waldgrenze; dort treten in den Tälern, zwischen kahlen Bergen, im allgemeinen zwergwüchsige
Bergbirken auf. Südlich dieser Zone, in Mittellappland und im Küstenbereich des Bottnischen Meerbusens, ist der Anteil der von der Kiefer beherrschten Wälder am größten. Im Unterholz wächst hier vor allem die Rentierflechte. Sie bildet die hauptsächlichste Nahrung der Rentiere, weshalb die trokkenen Nadelwälder für die Rentierzucht in Nordfinnland, vor allem in Lappland, große Bedeutung haben. Wälder, in denen die Fichte vorherrscht, gibt es unter anderen in Kainung und in Kuusamo.

Die Kargheit der Natur Nordfinnlands spiegelt sich im langsamen Wachstum der Bäume wieder. In der Provinz Oulu beträgt der Zuwachs ungefähr $2 \mathrm{~m}^{3}$ pro ha im Jahr, in Nordlappland weniger als $1 \mathrm{~m}^{3}$ pro ha im Jahr. In der Provinz Oulu benötigt eine Kiefer ungefähr 100 Jahre, in den Gebieten von Ylitornio und Rovaniemi 140-160 Jahre und im Gebiet von Inari 200 Jahre, um ihr volles Stammvolumen zu erreichen. Andererseits sind die Bäume in Nordfinnland auf Grund ihres langsamen Wachstums dichtfaserig und ihr Holz ist so fest, daß sie für viele $Z$ Zwecke wertvolles Rohmaterial liefern. 


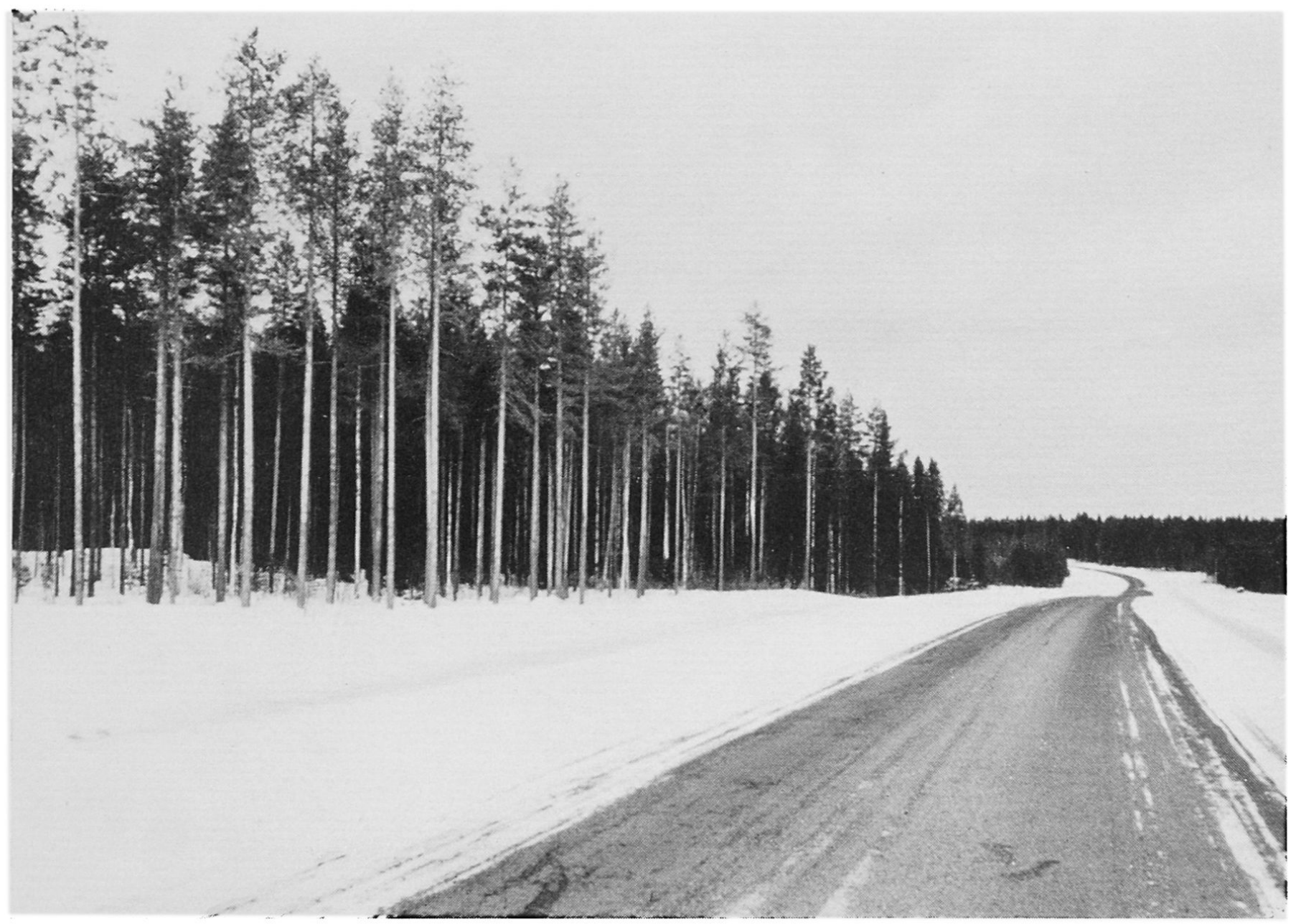

Abbildung 6. Straße zwischen Oulainen und Ylivieska. Die Straßen sind auch in Nordfinnland ziemlich gut. Photo M. Häkkilä

Nicht ganz Nordfinnland wird jedoch von Wald bedeckt; weite Flächen werden von Mooren eingenommen. Die Grenze zwischen Sümpfen und Wäldern ist nicht immer klar zu erkennen; in manchen Zonen, die eigentlich zu den Sümpfen gezählt werden, wachsen so zahlreiche Bäume, daß sie als Wälder anzusehen sind. Die finnischen Sümpfe werden in drei Haupttypen unterteilt: Sumpfbruchwald (finnisch «korpi»), Reisermoor (finnisch «räme») und offenes, baumloses Moor (finnisch «avosuo»). In den Sumpfbruchwäldern wächst ein ziemlich guter Fichten- oder Birkenwald. In den Reisermooren dagegen gedeiht als Holzart am besten die zwergwüchsige Kiefer. Die baumlosen Moore sind, wie schon ihr Name besagt, völlig ohne Baumbewuchs.

Sumpfbruchwälder kommen am häufigsten im Küstenbereich des Bottnischen Meerbusens, in Kainuu und in Westlappland vor: in diesen Gebieten beträgt ihr Anteil an der gesamten Sumpf- und Moorfläche 20-50\%. Den größten Teil der nordfinnischen Sümpfe bilden Reisermoore. Im Haupt- teil Nordfinnlands, mit Ausnahme von NordwestLappland, und in einem Teil von Kainuu beträgt ihr Anteil an der gesamten Sumpf- und Moorfläche mehr als $50 \%$. Offene, baumlose Moore findet man, vor allem im baumlosen Nordlappland und im mittleren Südteil der Provinz Lappland wie auch im Nordteil der Provinz Oulu.

Auch in Nordfinnland hat die natürliche Vegetation in weiten Gebieten dem Feld- und Grasbau weichen müssen. Die Landwirtschaft wird von der Viehzucht beherrscht, der größte Teil der landwirtschaftlichen Nutzfläche wird von Wiesen und Weiden eingenommen. Heugras gedeiht in Nordfinnland bis hoch nach Lappland genau so gut wie im Süden. Aber auch Getreide wird angebaut. Das Pflanzgebiet der Gerste erstreckt sich bis nach Nordlappland. Hafer wird noch in den südlichen Teilen der Provinz Lappland, Roggen ganz allgemein im Hauptteil der Provinz Oulu angepflanzt. Das Anbaugebiet der Kartoffeln erstreckt sich ebenfalls bis nach Nordlappland. 
Aario L. (1966): Suomen maantiede. Helsinki. Atlas of Finnland. Helsinki 1960.

Helle R. (1966): An Investigation of Reindeer Husbandry in Finland. Acta Lapponica Fenniae 5.

- (1970): A Study of Tourism in Lapland. Nordia 1970, 2.

Hult J. (1966): The Areal Differentiation of Farming in the Oulu District, Finland. Fennia 87; 1.

Ilvessalo Y. (1960): Suomen metsät kartakkeiden valossa. Deutsches Referat: Die Wälder Finnlands im Licht von Karten. Communicationes Instituti Forestalis Fenniae 52; 2.

Kalela A. (1958): Über die Waldvegetationszonen Finnlands. Botaniska Notiser 111; 1,
Naukkarinen A. (1969): Population Development in Northern Finland 1950-1965. Nordia 1969, Nummer 8.

Ruuhijärvi R. (1960): Über die regionale Einteilung der nordfinnischen Moore. Annales Botanici Societatis Zoologicae-Botanicae Fennicae "Vanamo», Tom. 31; 1 .

Suomenmaa. Maantieteellis-yhteiskunnallinen tietoja hakutes. Osa I. Porvoo 1967.

Suomen tilastollinen vuosikirja 1968. (Statistical Yearbook of Finland 1968).

Suomi. A General Handbook on the Geography of Finland. Published by the Geographical Society of Finland. Fennia 72.

Varjo U. (1967): Lapin maatalous 1950-59. Lapin seutusuunnittelun kuntainliitto. Rovaniemi.

- (1966): Über die Straßen Finnlands. Fenni 92; 6. 